\title{
Flaring stellar disk in the low surface brightness galaxy UGC 7321 (Corrigendum)
}

\author{
S. Sarkar and C. J. Jog
}

\author{
Department of Physics, Indian Institute of Science, Bangalore 560012, India \\ e-mail: suchira@iisc.ac.in, cjjog@iisc.ac.in
}

A\&A 628, A58 (2019), https://doi.org/10.1051/0004-6361/201935430

Key words. galaxies: halos - galaxies: ISM - galaxies: individual: UGC 7321 - galaxies: kinematics and dynamics galaxies: spiral - errata, addenda

This is a corrigendum for Sarkar \& Jog (2019). A negative sign was inadvertently placed in front of the surface brightness values plotted along the $Y$-axis in Figs. 9-11. The actual values of surface brightness calculated using Eq. (10) of the paper are positive, and hence the intensity falls at high $z$, as expected physically. There is no change in the results or conclusions of the paper.
We thank Dmitry Bizyaev for drawing our attention to this mistake.

\section{References}

Sarkar, S., \& Jog, C. J. 2019, A\&A, 628, A58 